\title{
Implementation and Analysis of Various PAPR Reduction Techniques using MATLAB
}

\author{
Ankit Bass \\ Student, SECE \\ Lovely Professional University \\ Jalandhar, INDIA
}

\author{
Sudipta Ghosh \\ Student Member IEEE \\ Lovely Professional University \\ Jalandhar, INDIA
}

\author{
Vikas Singh \\ Student, SCS \\ Lovely Professional University \\ Jalandhar, INDIA
}

\begin{abstract}
Orthogonal Frequency Division Multiplexing is a new and emerging technology that provides us with high data rate. But every coin has two sides, pros and cons. So does OFDM. On the better side it provides us with high data rate but on the down side we have PAPR. The main obstacle for OFDM to work more efficiently is PAPR i.e. peak to average power ratio which is nothing but several sinusoidal leads. The purpose of this paper is to provide a MATLAB simulation of PAPR using some reduction techniques so that better performance could be achieved.
\end{abstract}

\section{General Terms}

Fast fourier transform (FFT), Inverse fourier Transform (IFFT), Pulse Shaping, Filters

\section{.Keywords}

Orthogonal Frequency Division Multiplexing (OFDM),Peak Average to Power Ratio(PAPR), Systematic Odd Parity Checking Coding(SOPC), Power Amplifier(PA).

\section{INTRODUCTION}

Orthogonal frequency-division multiplexing (OFDM) is the modulation technique for European standards such as the Digital Audio Broadcasting (DAB) and the Digital Video Broadcasting (DVB) systems. As such it has received much attention and has been proposed for many other applications, including local area networks and personal communication systems. OFDM is a type of multichannel modulation [9] that divides a given channel into many parallel sub channels or subcarriers, so that multiple symbols are sent in parallel. Multiuser systems that use OFDM must be extended with a proper multiple-access scheme as must single carrier transmission systems. Compared to single carrier systems, OFDM is a versatile modulation scheme for multiple access systems in that it intrinsically facilitates both time-division multiple access and frequency-division multiple access . The communication track must be linear for OFDM to work properly and that too within the dynamic range that fits the PAPR distribution. Usually there is a problem with a linear Power Amplifier (PA) due to fact that its power efficiency is upper bounded and this upper bound decreases as the PAPR (the dynamic range of the PA) increases. This is the main reason why many PAPR reduction schemes have been proposed.

\section{ORTHOGONAL FREQUENCY DIVISION MULTIPLEXING (OFDM)}

OFDM is a Multicarrier Transmission technique which divides the available spectrum into many carriers each one being modulated by a low data rate stream. OFDM is similar to Frequency Division Multiple Access (FDMA) in that the multiple user access is achieved by sub-dividing the available bandwidth into multiple channels, which are then allocated to users. However OFDM uses the spectrum much more efficiently by spacing the channels more closer together. This is achieved by making all the carriers orthogonal to one another, preventing interference between the closely spaced channels. [6]

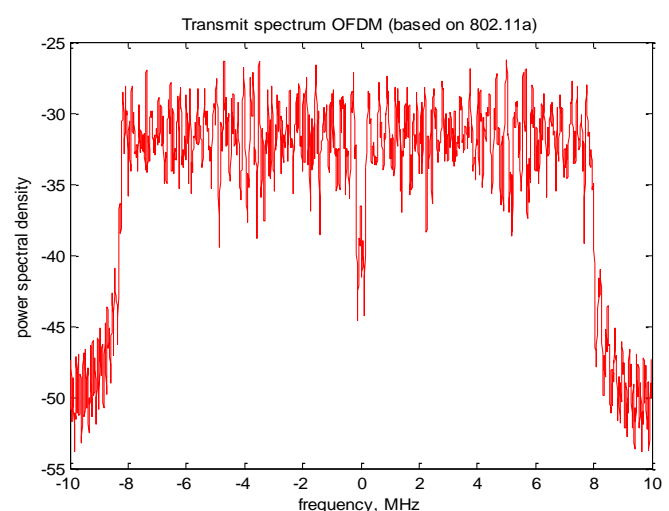

Figure 1. Normalized power spectrum of an OFDM signal

\section{Peak To Average Power Ratio (PAPR)}

The output of the OFDM modulator is given by:

$S\left(t_{-} x\right)=\sum_{n=0}^{N-1}\left|a_{n}\right| \cos \left[\left(\omega_{0}+n \Delta \omega\right) t+\phi_{n}\right], \quad 0 \leq t \leq T$

where $a=a_{0}, \ldots . ., a_{n-1}$ and $a_{n}=\left|a_{n}\right| e^{j \varphi_{n}}, \mathrm{n}=0,1,2$

3......., N-1. All symbols $a_{n}, n=0,1,2, \ldots \ldots . N-1$ are taken from constellation $\left\{a^{(1)}, a^{(2)}, \ldots . ., a^{(M)}\right\}$. Let,

$$
A_{\max }=\max _{1 \leq m \leq M}\left|a^{(m)}\right|
$$


The instantaneous (per symbol) peak to average power ratio is defined as:

$$
r(a)=\lim _{0 \leq t \leq T}|s(t, a)|^{2} / E\left\{\frac{1}{T} \int_{0}^{T}|s(t, a)|^{2} d t\right\}
$$

The IPAPR is a random variable and its distribution, for large $N$ is given by:

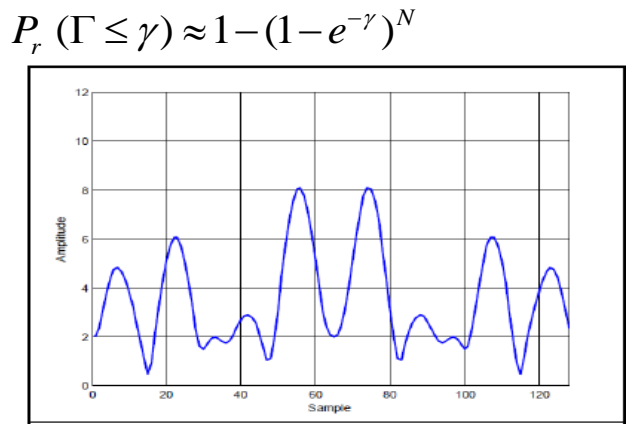

Figure 2: OFDM signal in time domain

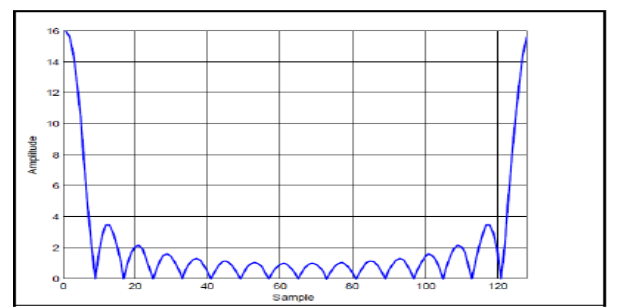

Figure 3: High PAPR when sub carriers are modulated by the same carrier

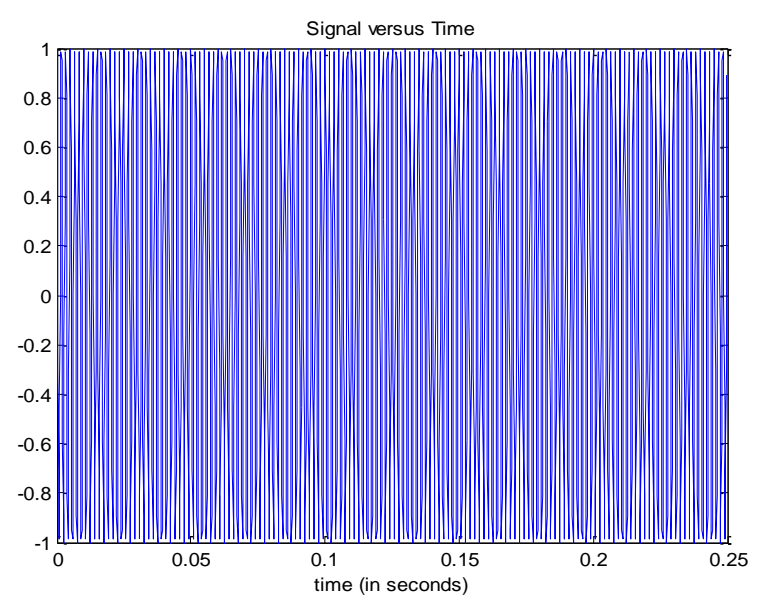

Figure 4: A sinusoidal signal with high peaks

Signal is transmitted with 64 data symbol per frame. This is the simulation of OFDM system to observe PAPR in it. Hence this is the explanation regarding PAPR and why it is reduce in OFDM system. There are different techniques that have been proposed for the reducing of PAPR in OFDM system [4].

\section{Techniques to reduce PAPR}

\subsection{Classic Clipping Technique}

In this technique, the signal having amplitude greater than a predefined threshold level A is limited to that level i.e. A and signal having amplitude lower than A are passed through undisturbed. The clipping function for this technique is defined as:

$$
f_{c}\left(r_{n}\right)=\left\{\begin{array}{lll}
r_{n} & \text { if } \mathrm{r}_{n} \leq \mathrm{A} \\
A & \text { if } \mathrm{r}_{n}>\mathrm{A}
\end{array}\right.
$$

\subsection{Deep Clipping Technique}

Deep clipping method is used suppress the peak re-growth to reduce the PAPR of OFDM signals. The clipping function is modified in order to "deeply" clip the high amplitude peaks. A parameter called clipping depth factor has been introduced in order to control the depth of the clipping. The function based clipping used for this technique is defined as:

$$
f_{c}\left(r_{n}\right)=\left\{\begin{array}{l}
r_{n} \text { if } \mathrm{r}_{n} \leq \mathrm{A} \\
A-\alpha\left(r_{n}-A\right) \text { if } \mathrm{A} \backslash \mathrm{r}_{n} \leq \frac{(1+\alpha) A}{\alpha}
\end{array}\right.
$$

\subsection{Smooth Clipping Technique}

A Smooth Clipping technique used to reduce the OFDM PAPR is described by the function based-clipping for this technique is defined as:

$$
f_{c}\left(r_{n}\right)=\left\{\begin{array}{l}
r_{n}-\frac{r_{n}^{3}}{b} \text { if } \mathrm{r}_{n} \leq 3 \mathrm{~A} / 2 \\
A \text { if } \mathrm{r}_{n}>3 \mathrm{~A} / 2, \text { where } \mathrm{b}=\frac{27 \mathrm{~A}^{2}}{4}
\end{array}\right.
$$

\subsection{PAPR Reduction by Clipping}

Suppose the OFDM signal is clipped at a level th yielding a PAPR that is less than or equal to $\gamma t h$. The probability of error is given by : $P b=\sim$ PePclip $+P e(1-P$ clip $)$, [8] where Pclip $=\operatorname{Pr}(\Gamma>\gamma t h), \sim P e$ is the probability of error when the symbol is clipped and $P e$ denotes the probability of error for an unclipped symbol. Pclip depends on $\gamma$ th and, increases as $\gamma$ th decreases. ${ }^{\sim} P e$ depends on both $\gamma t h$ and $S N R$, but usually the influence of $\gamma$ th is a dominant one and $\sim P e$ increases as $\gamma$ th decreases. $P e$ depends only on $S N R$ and therefore $P b$ increases as $\gamma t h$ decreases and therefore $\alpha<0$ [4]. 


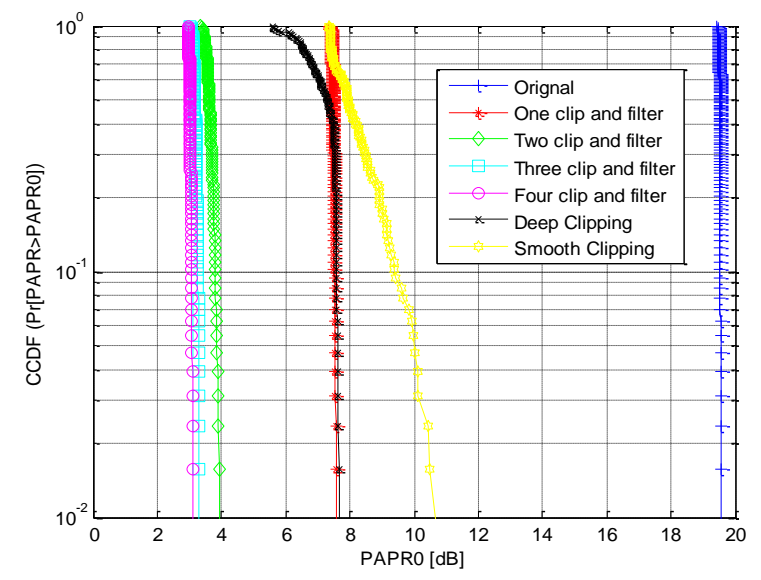

Figure 5. Signal obtained in logarithmic scale after using the various clipping techniques

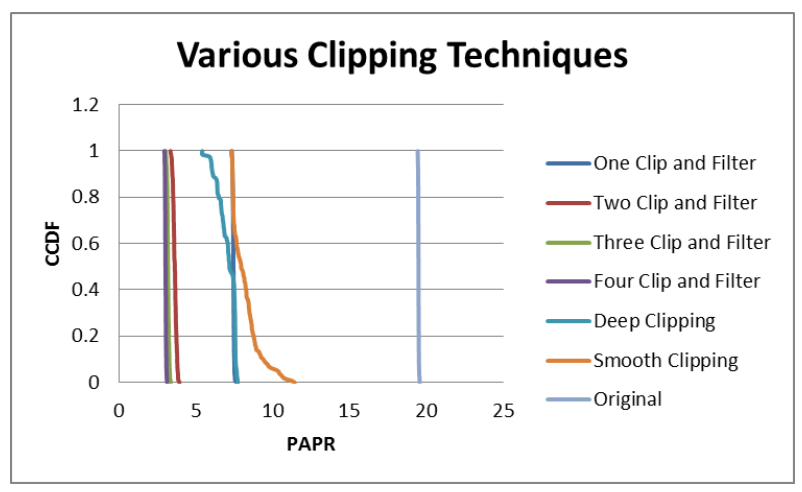

Figure 6. Signal obtained after using the various clipping techniques and

\subsection{PAPR Reduction by Tone Injection}

This method is generally used additive method for PAPR reduction. Using this method data rate loss is very less. This method used the set of active constellation point for an original constellation point to reduce the PAPR. in this each unit all original constellation is mapped on the several equivalent constellation point. \& this extra points freedom can be easily used to reduce the PAPR. This method is popularly used as the tone injection method because of the newly applying points into basic constellation for the new points for larger constellation. Main thing is injecting tone of appropriate phase and frequency in OFDM symbol. Main demerits are that transmission of side information is necessary at the receiver side.

\subsection{Envelope Scaling}

This technique is related to scaling means before OFDM signals sent to the IFFT all subcarrier is scaled the input envelope. in this technique 256 subcarrier is used so all subcarrier will remains equal. Main idea is that to scheme is that the input envelope in some sub carrier is scaled to achieve the smallest amount of PAPR at the output of the IFFT. Here receiver does not need any side information at the receiver end for decoding. This scheme is suitable for the PSK modulation .when it is applied with the QAM high degradation is occurred in the BER.

\section{CONCLUSION}

According to the information regarding all above described techniques to reduce the PAPR in OFDM system all techniques are different in their way and by using each technique PAPR can be reduced to some what level. The efficient PAPR exists for any PAPR reduction technique for which the following statement is true the BER of the information bits increases as the reduced PAPR decreases.

\section{REFERENCES}

[1] X. Li and L. J. Cimini, "Effects of clipping and filtering on the performance of OFDM," IEEE Commun. Lett., vol. 2, pp. 131-133, May 1998.

[2] D. Wulich, N. Dinur, and A. Gilinowiecki, "Level clipped high order OFDM," IEEE Trans. Commun., vol. 48, pp. 928-930, June 2000.

[3] A. R. S. Bahai, M. Singh, A. J. Goldsmith, and B. R. Saltzberg, "A new approach for evaluating clipping distortion in multicarrier systems," IEEE J. Select. Areas Commun., vol. 20, pp. 3-11, May 2002.

[4] J. Tellado, Multicarrier Modulation with Low PAR: Applications to DSL and Wireless. Kluwer Academic Press, 2000.

[6] Mohammad Zavid Parvez Md. Abdullah Al Baki "Peak To Average Power Ratio (Papr) Reduction In Ofdm Based Radio Systems", Master Of Science Thesis Blekinge Institute Of Technology May 2010.

[7] P. Boonsrimuang, E. Puttawong, H. Kobayashi and T. Paungma, "PAPR Reduction Using smooth clipping in OFDM system", 3rd Information andComputer Engineering Postgraduate Workshop, pp. 158-161, 2003.

[8] Ali N. Akansu, Pierre Duhamel, Xueming Lin, and Marc de Courville, "Orthogonal transmultiplexers in communication: A Review", IEEE Transactions on Signal Processing, Vol. 46, No. 4, pp. 979-995, 1998.

[9] Sudipta Ghosh, Ankit Bass, "Implementation of digital video broadcasting terrestrial (DVB-T) using orthogonal frequency division multiplexing (OFDM) on physical media dependent sub layer", IJCA (International Journal of Computer Application), Volume 44, number 22,April 2012, Pg 20 to 25 